\title{
Design and Assemble of Low Cost Prepaid Smart Card Energy Meter - A Novel Design
}

\author{
Sudarshan K. Valluru \\ Department of Electrical Engineering, Delhi Technological University \\ (Formerly Delhi College of Engineering) \\ Bawana Road, Delhi-110042, India. \\ valluru.sk@gmail.com.
}

\begin{abstract}
In India, presently distribution companies are collecting revenues from their consumers by metering and billing. The expenses for metering, billing, and collecting dues became complex as electricity is supplied to large number of consumers located in far-flung areas, resulted in high cost per unit. This may encourage consumers to fudge meter readings. To address these problems, distribution companies can opt prepaid smart card energy meter, which overcomes the whole problems associated with the present billing system and will make the consumer more conscious about usage electricity. This paper explores the design and assemble of smart card prepaid electricity meter system, which enables the consumer to buy required amount of energy and also provides information about their credit balance. A prototype of the system has been developed with the state-of-the-art digital and information technology and proven to be stable, reliable, tamper resistant, low cost, and easy maintenance.
\end{abstract}

Keywords: Smart Card, Micro controller AT89C51, MAX-232 Serial Communication, Liquid Crystal Display, Pre-paid Electricity Protocol

\section{Introduction}

Electricity is a major source of energy in the world and used in every civilized country for domestic, commercial and industrial purpose. Recently owing to raise in number of new housing, commercial and industrial developments in India, the number of consumers of electricity have been increased in the network distribution. The monthly power bill for consumers is calculated from post-paid meter reading based on the electricity consumption. But the disadvantage of this system is that meter readers are needed to visit every door to door to read the meter for billing purposes. This is a tiresome, lengthy and error prone process. Moreover, there is a chance for consumers as well as meter readers to do the corruption. In post-paid system [1], the monthly bill sent to the consumer by mail in the beginning of the month and all the consumers often need to gather in a long queue to pay the bills. This procedure is tiresome and time consuming.

In this paper, a prepaid metering (PPM) system [2] has been proposed. Each consumer has to install digitally designed prepaid electricity meter along with smart card in their home, office or industry. Based on the electricity utilization the credit will be deducted automatically from the smart card. When the credit goes below the threshold value, the meter gives warning icons and if credit reaches to zero the meter will automatically cut off the power supply. The consumer has to go to the vending station with the smart card and money to buy electricity. In the vending station, user can buy the credit to his smart card by paying the money. Then the consumer will insert the smart card to the meter and the meter will be recharged. In prepaid billing system, the consumer will be always conscious about the remaining credit and thus try to use electricity carefully and stop its misuse. Furthermore, in contrast with post-paid billing, the consumer can buy credit at any time in the month from the vending station.

Received: August $31^{\text {st }}$, 2013. Accepted: February $3^{\text {rd }}, 2014$ 
In the present proposed scheme there is no need of manual intervention to note meter reading which avoids hassle in billing and distributions companies make money promptly which certainly helps them to generate more revenue.

\section{PPM System Architecture}

Prepaid meters (PPM) will be installed in home, commercial or industries. For a specific region there will be a vending station also called cash dispensing unit (CDU) from where the consumer will buy credit. The vending stations hold the regional user data base and billing software. The communication between the meter and the vending station is done by smart card. All vending stations are connected with a central server called the master station through the Internet. Master station holds the integrated database of all the vending stations, user account creation software and overall system analysis tools. The overall architecture of the prepaid metering system is shown in Figure1.and brief description of several components of the system is discussed below.

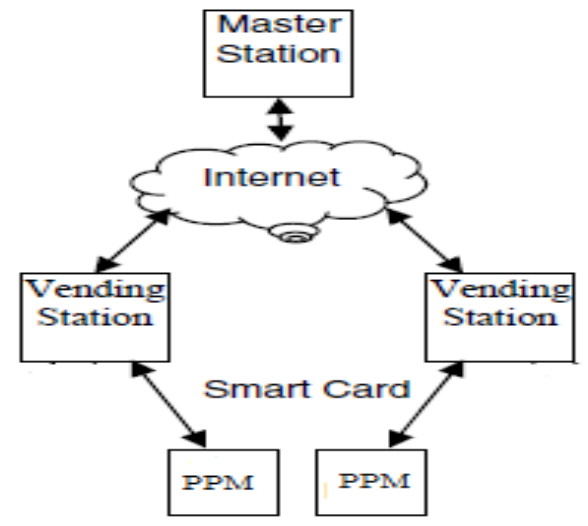

Figure 1. Architecture of the Prepaid Metering System

\section{A. Master Station}

Master station holds the integrated database of all the vending stations. Creation, edition and deletion of users account are also done from here. In the master station, the values of the meter settings variables are given. User transaction report or meter status report of any user can be generated from here. As the master station holds the integrated database of all the vending stations, the overall analysis of the system can be done from here.

\section{B. Vending Station}

Vending station is the office where the clients go to by credits and holds the database of all local users. Every user of the prepaid meter has smartcards, which establishes the connectivity between the PPM and the vending station. The Figure 2. shows the basic components of vending station mainly consist of server PC, smartcard reader/writer and slip printers to print receipts. When a client needs to buy credit, goes to the local vending station with the smart card and money. To keep the database of the vending station up-to- date, clients are advised to punch the smart card in the meter before coming to the vending station. When the card is punched, the meter's last status is copied to the smart card. In the vending station, the last status of the meter is firstly read from the smart card and save it in database. Then according to the client's demand, vending station writes the credit in the smart card. When the user returns home and punch the smart card in the meter, the credit is added with the remaining credit and the new meter settings are activated. Vending stations maintain relation with the master station through internet. Vending station uploads all the meter status and vending data to the master station and it downloads the latest meter settings from the master station. 


\section{Smart card Unit}

A smart card reader/writer [3] is interfaced using the serial three wire bus protocol with the CPU to read/write data to smart card. The smart card reader's Reset and Clock pins are connected with two output ports of the CPU. The Data pin is connected with a port which can be configured both in input and output direction so that data can both be read and write to smart card. The SLE4428 type smart card has been used with $1 \mathrm{~KB}$ of non-volatile EEPROM memory and it is write-protected by a 16 bit password. Different meter settings and meter status variables are mapped in different memory locations of the smart card.

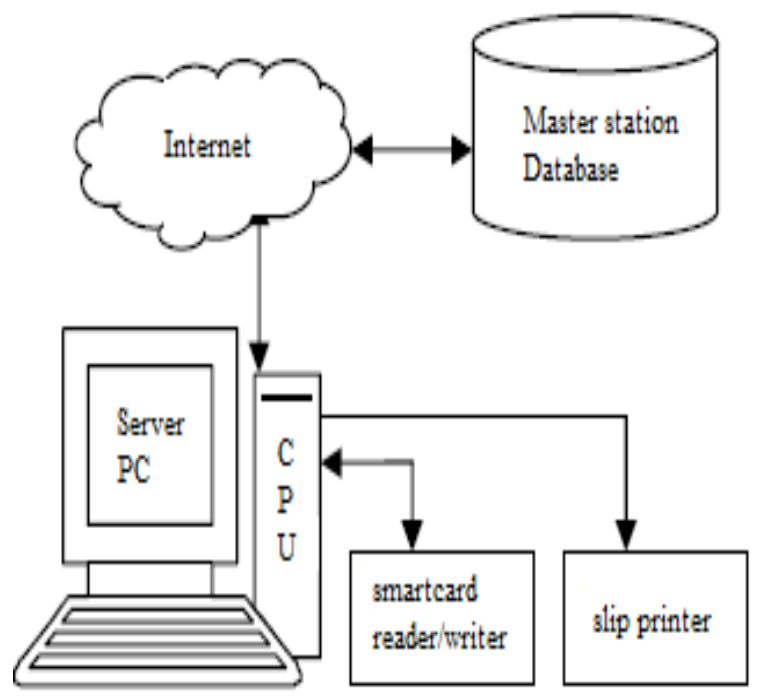

Figure 2. Block diagram of Vending Station

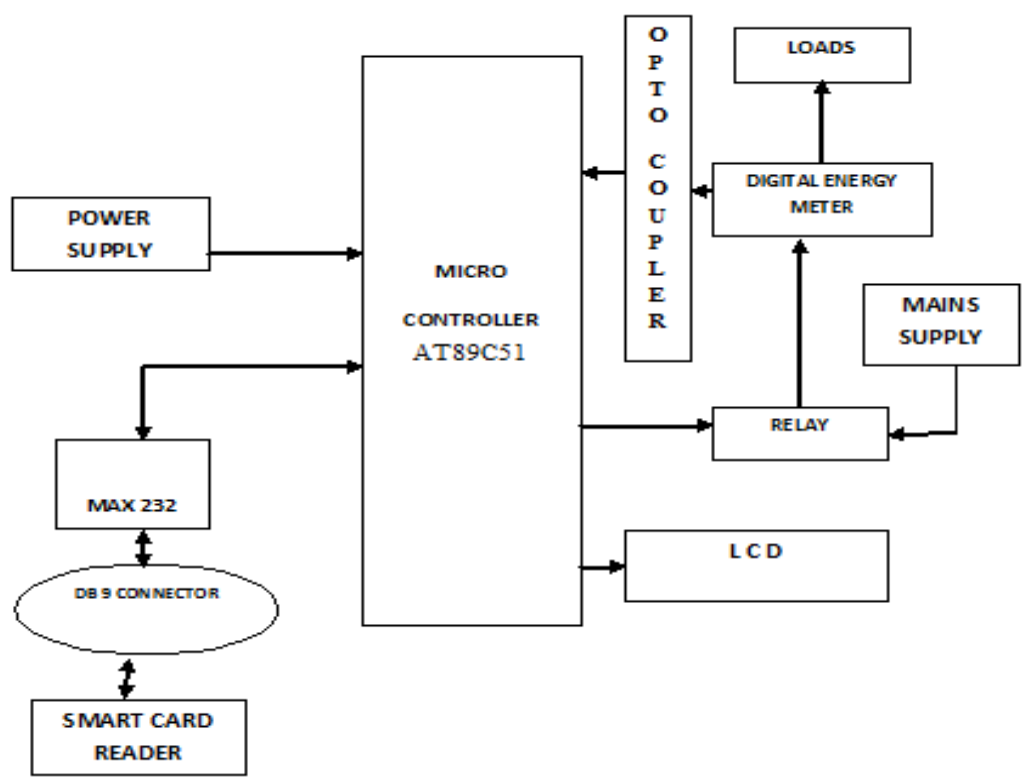

Figure 3. Block diagram of Prepaid Energy Meter 


\section{Prepaid meter}

The most important constraint is design and assembles of prepaid energy meter. The block diagram of prepaid energy meter [4] is shown in Figure3.and its components are discussed below sections.

\section{The Design of Intelligent Controller}

This system is composed of software and hardware structures. The hardware structure consists of a digital energy meter module interfaced to an AT89C51 [5-11] low-power, highperformance CMOS 8-bit micro controller has 40-pin, a highly stable $24 \mathrm{MHz}$ oscillator, $4 \mathrm{~KB}$ flash programmable and erasable read only memory (PEROM), 128 bytes of RAM, 32 I/O lines, two 16-bit timer/counters, a five vector two-level interrupt architecture, a full duplex serial port, on-chip oscillator and clock circuitry. In addition, the AT89C51 is designed with static logic for operation down to zero frequency and supports two software selectable power saving modes. The Idle Mode stops the CPU while allowing the RAM, timer/counters, serial port and interrupt system to continue functioning. The Power-down Mode saves the RAM contents but freezes the oscillator disabling all other chip functions until the next hardware reset.PIN P0.0-P0.7, P1.0- 1.7, P2.0 - P2.7, and P3.0-P3.7 may as general I/O and LCD analog output. In the16pin LCD, first pin grounded, Second pin connected to power supply and $3^{\text {rd }}$ pin will be connected to variable pot to control the contrast of the LCD. LCD data lines are connected to port P0, The microcontroller pins P2.7, P2.6 and P2.5 are connected to the LCD control pins RS, R/W and EN respectively. The ALE, PSEN, EA pins are left isolated. For the execution of the program of micro-controller requires clock pulses of frequency $12 \mathrm{MHz}$ which generated by the crystal oscillator with grounding capacitance 33PF,therefore XTAL2 and XTAL1 pins of micro-controller are connected to the crystal oscillator. The RX(P3.0) and TX (P3.1) are connected to the 12 and 11 pins of MAX 232 serial communication via DB9 connector to interface the Smart Card Reader module, from that smart card the data regarding the amount is fed to the micro controller gets activated and starts to take the data from the 4N35 optocoupler. The micro controller P3.2 connected to the IRLED digital energy meter via optocoupler, with external interrupt0 the number units consumed is counted with the help of the counted pulses which are generated from the optocoupler. The IRLED blinks when there is a unit consumed gives the IR rays incident on optocoupler which itself drives the transistor base connected to P3.2. The micro-controller counts the number of units and simultaneously monitors the smart card reader for the presence of the card. The micro-controller reads the total purchased units from the smart card and unit's value has been reduced by one for each and every unit consumed. If the units in the smart card become zero then the micro-controller gives the signal to the relay to switch off the total supply to the energy meter. The P2.0 is connected to the relay through the transistor. The $40^{\text {th }}$ pin $\left(\mathrm{V}_{\mathrm{cc}}\right)$ and $20^{\text {th }}$ pin (GND) of microcontroller connected to $5 \mathrm{~V}$ power supply and ground respectively. $9^{\text {th }}$ pin will be connected to the reset and by pressing the reset pin the C-Language program in the micro-controller will be executed. Therefore the micro controller gets the data from the energy meter and SLE4428 card reader and also controls the total power supply of the system [12]. The design diagram of intelligent AT89C51micro controller, energy meter, Display and Relay Control Circuit is shown in Figure 4. 


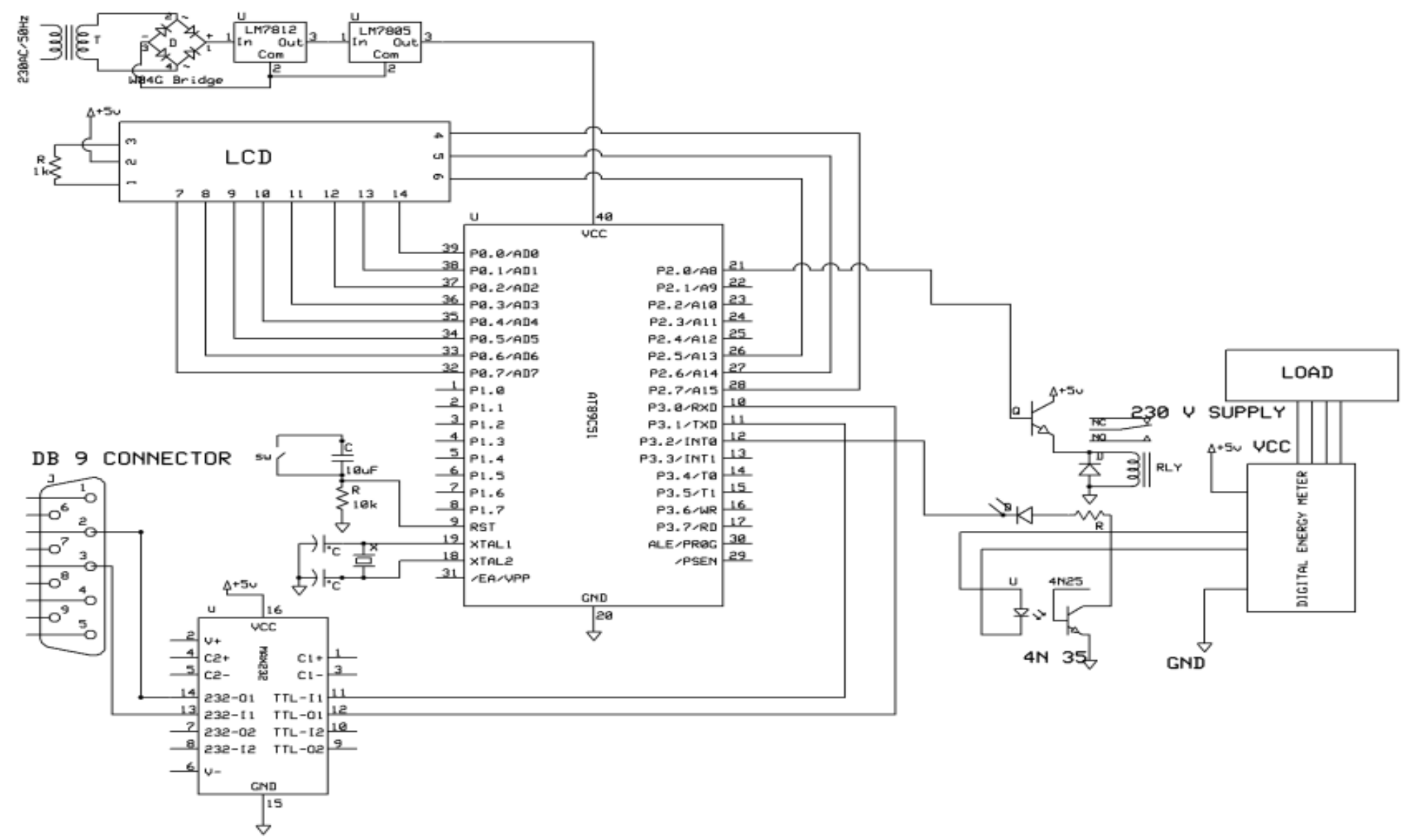

Figure 4. Display and Relay Control Circuit of Prepaid Energy Meter 


\section{A. The Design of Software}

The main protocol has five phases for controlling the consumption of electricity based on proper authorisation by using smartcard; balance checking, decision making, decision process and termination. Control systems for working process main protocol [13] flow chart is shown in Figure5.and the other sub-modules of flow chart are omitted.

\section{B. Prototype Development}

Based on the design discussed above, prepaid smart card energy meter shown in Figure6 has been constructed and assembled in the laboratory; the meter technical specifications [14] are shown in Table 1. The test results are tabulated in Table 2 and precision experimentally obtained for this new meter better than $97 \%$. This value is compatible with the commercially available energy meters in the market.

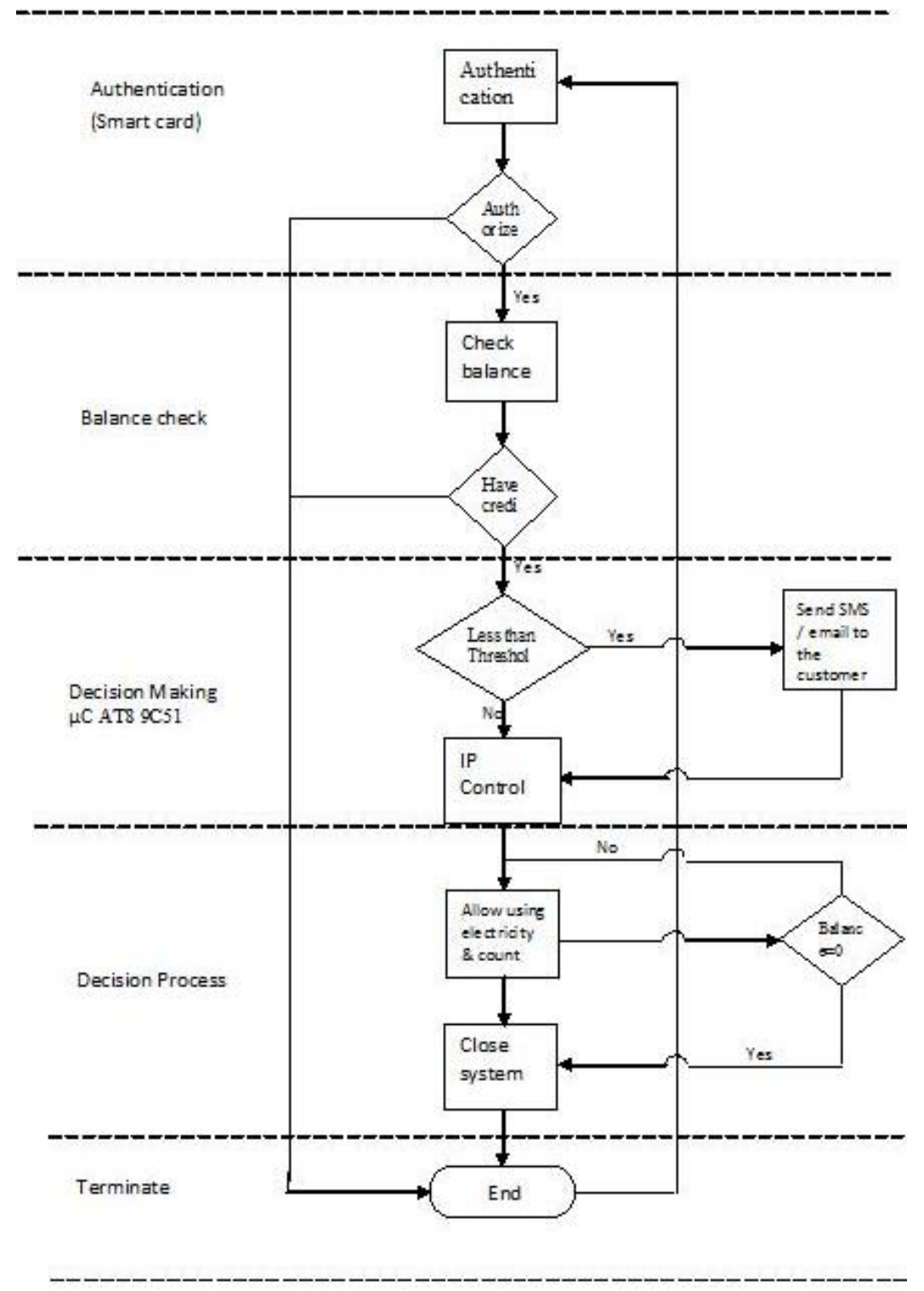

Figure 5. Pre-paid electricity protocol 


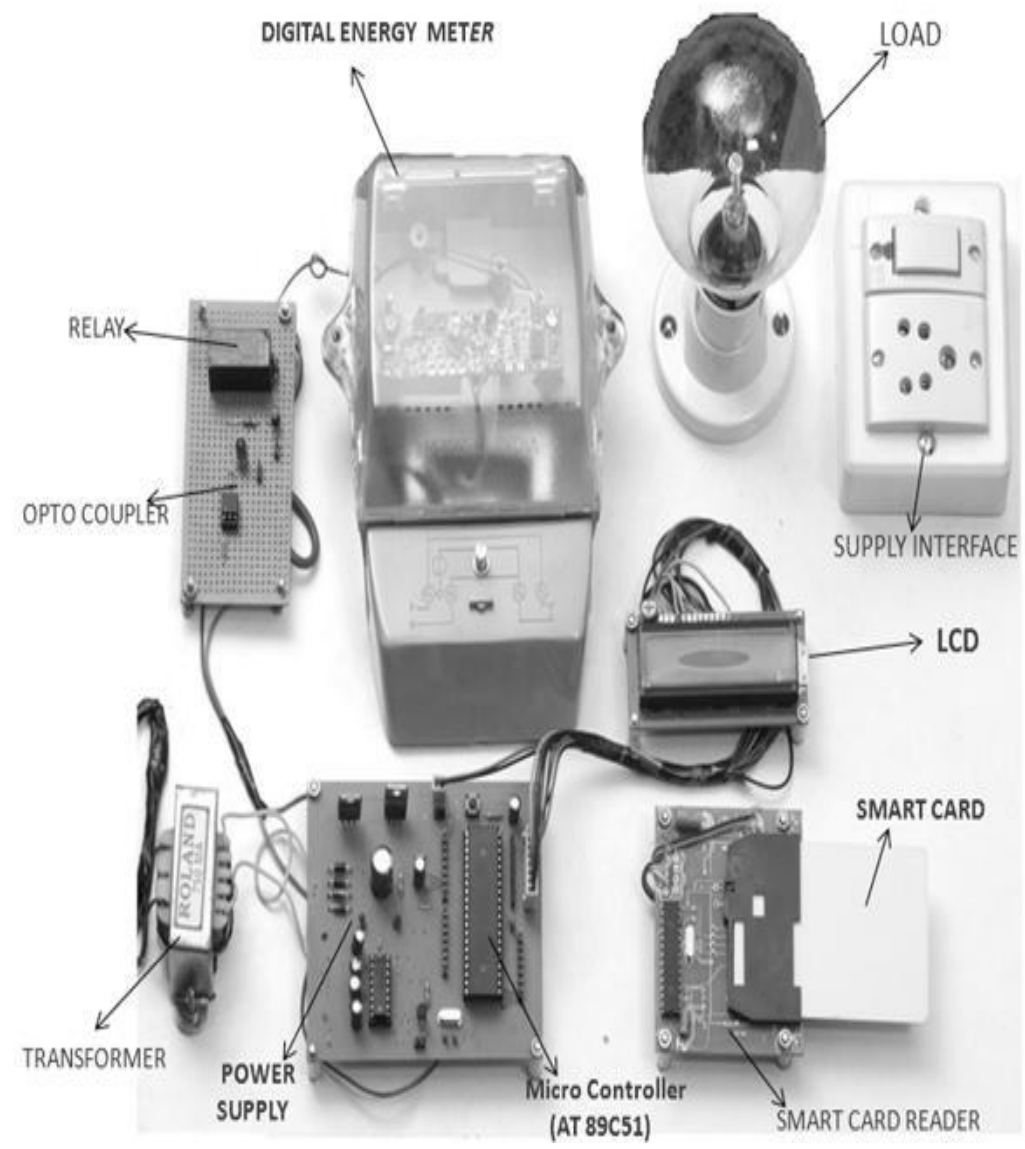

Figure 6. Proto type of Pre-paid smart card meter

Table 1.Technical Specifications

\begin{tabular}{|c|c|c|c|c|c|}
\hline Type & Accuracy & $\begin{array}{c}\text { Rated } \\
\text { Voltage }\end{array}$ & Frequency & Display & Information Record \\
\hline 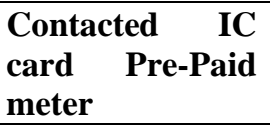 & Class 1.0 & $\begin{array}{l}\text { Single- } \\
\text { Phase } \\
230 \mathrm{~V}\end{array}$ & $50 \mathrm{~Hz}$ & $\begin{array}{l}\text { LED } \\
\text { display }\end{array}$ & $\begin{array}{l}\text { The energy credited } \\
\text { and used will be } \\
\text { recorded }\end{array}$ \\
\hline
\end{tabular}

Table 2. Test results over 24 - hours

\begin{tabular}{|l|c|c|c|c|c|c|}
\hline $\begin{array}{c}\text { Lamp } \\
\text { load(W) }\end{array}$ & $\begin{array}{c}\text { Number } \\
\text { of } \\
\text { Hours }\end{array}$ & $\begin{array}{c}\text { Load } \\
\text { Measured } \\
\text { by Standard } \\
\text { Meter(kWH) }\end{array}$ & $\begin{array}{c}\text { Load } \\
\text { Measured } \\
\text { by Pre-Paid } \\
\text { Meter(kWH) }\end{array}$ & Deviation & $\begin{array}{c}\text { Error } \\
\mathbf{( \% )}\end{array}$ & $\begin{array}{c}\text { Precision } \\
\mathbf{( \% )}\end{array}$ \\
\hline $\mathbf{2 0 0}$ & 6 & 1.2 & 1.17 & 0.03 & 2.5 & 97.5 \\
\hline $\mathbf{1 0 0}$ & 6 & 0.6 & 0.582 & 0.018 & 3.0 & 97 \\
\hline $\mathbf{6 0}$ & 6 & 0.36 & 0.3528 & 0.0072 & 2.0 & 98 \\
\hline $\mathbf{4 0}$ & 6 & 0.24 & 0.2328 & 0.0072 & 3.0 & 97 \\
\hline Mean & 24 & 2.4 & 2.3376 & 0.0624 & 2.6 & 97.4 \\
\hline
\end{tabular}




\section{Conclusion}

A prototype of the system has been developed and tested successfully. In this paper, the advantages of prepaid metering system and its design components have been discussed. The meter features includes with alarming of remainder, storing data up to 20 years. This prepaid metering system minimizes the human intervention in meter reading, bill calculations and bill delivery which ultimately reduces many defects than the currently existing post paid billing systems. This system was specially designed for India but can also be implemented in any other country. Of course, the basic principles implemented here can be easily extended to the case of three-phase meters. This is certainly beneficial to both consumers and power distribution companies.

\section{References}

[1] M I Tibbenham, "Implementing a Pre-payment System” IEE International Congress on Metering and Tariffs for Energy Supply, Birmingham, UK, 1999, pp 251-257.

[2] Muhammad Faheem Khan, Ahmed Zoha, and Rana Liaqat Ali ''Design and Implementation of Smart Billing and Automated Meter Reading System for Utility Gas"' Proc. in IEEE Conference on Information and Emerging Technologies, 2007, pp1-6

[3] KwanB H, M Moghawemi 'PIC Based Smart Card Prepayment System' ' Proc. in IEEE Student Conference on Research and Development, Malaysia,2002, pp 440-443

[4] PAV. Loss, MM. Lamego, GCD. Sousa and JLF.Vieira, "A Single Phase Microcontroller Based Energy Meter", Proc. in IEEE Instrumentation and Measurement, Technology Conference, Minnesota, 1998, pp 797-800.

[5] Huang Ji-wu, Yang Hong-wei, Ming Feng " Study on an Application Using AT89c51 to Measure Frequency in UHF and VHF”, Wuhan University Journal of Natural Sciences, Vol 48 (1), 2002, pp 125-128.

[6] Fuquan Pan, Lixia Zhang, Gang Sun, Jiyou Li, “ Design of Vehicle Reversing Collision Avoidance Device based on Single Chip Computer" $2^{\text {nd }}$ International Conference on Power Electronics and Intelligent Transportation System (PEITS), Shenzhen, 2009, pp. 223-226.

[7] Wei Shutao, Chen Lin, Cui Lili and Wang Zhenxing "Interface Design of EEPROM and Signle Chip Micro Computer by IIC Bus" International Conference on Information Management and Engineering, Kula Lumpur, 2009, pp 554-557.

[8] Jishun Jiang, Lina Liu, Tian Jiang "Study of A New Type Field Irrigation Intelligent Controller" Proc in $2^{\text {nd }}$ IEEE International Conf. on Information Engineering and Computer science, 2010, pp1-4.

[9] Wang Yuanzhe, Bai Liyuan, "Electric Cable Interface Temperature Monitoring and Early Warning System" Second WRI Global Congress on Intelligent Systems, Wuhan, 2010, pp. 211-215.

[10] Li Xiangyang, Luo Genghe, Yan Jie, Guo Yan Ling, “The communication interface design of AT89C51 preparation robot based on Serial" International Conference on Electric Information and Control Engineering, Wuhan, 2011, pp 802-804.

[11] Zhu Weiguo, Ruan Hailin "Design and Research of Solar Photovoltaic Power Generation Controller based on 89C51 microcontroller” Advance Materials Research Vol. 345, 2012, pp. 66-69.

[12] Tian Yew Lim and Tat-Wai Chan "Experimenting Remote Kilowatthour Meter Reading Through Low-Voltage Power Lines at Dense Housing Estates" IEEE Transactions on Power Delivery, Vol. 17, No. 3, July 2002. pp. 708-711

[13] Ross J. Anderson, S.Johann Bezuidenhout, "Cryptographic Credit Control in Prepayment Metering Systems" Proc. in IEEE Symposium on Security and Privacy, Oakland,USA, 1995 pp15-22.

[14] Manual on Standardization of AC Static Electrical Energy Meters, Publication No: 304, Central Board of Irrigation and Power, New Delhi , 2008 


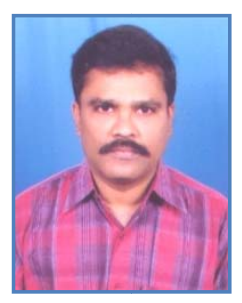

Sudarshan K. Valluru is working as Associate Professor in Electrical Engineering department of Delhi Technological University (Formerly Delhi College of Engineering), Govt. of NCT of Delhi and has 18 years of academic experience. He received his B.Tech in Electrical and Electronics Engineering from Nagarjuna University, Andhrapradesh and M.Tech in Computer Applications in Industrial Drives from National Institute of Engineering, Mysore, Karnataka. So far he has published 4 papers in international and 1 in national referred journals, 2 papers in IEEE International conferences and 5 in national conferences. He is authored a book on Neural Networks, Fuzzy Logic and Genetic Algorithms (Jaico publishers 2010). His areas of interest are AI systems, Instrumentation and Control. He is a senior fellow member of Institution of Electronics and Telecommunication Engineers India, Member in Indian Society for Technical Education India, Institution of Engineers India and IEEE. 